\title{
SUPER RESOLUTION RECONSTRUCTION OF IMAGE GRADIENT PROFILE SHARPNESS
}

\author{
Minal Chandurkar ${ }^{1}$ and Roshni Khedgaonkar ${ }^{2}$
}

\begin{abstract}
Single Image super resolution is an active and classic image processing problem, which aims to reconstruct a high resolution (HR) single image from a low resolution input image. Due to the several use of profile dictionary under-determined nature of this type of problem, an effective image prior is necessary to make the problem solvable, and to improve the quality of reconstruct super resoluted image. In this paper image super resolution algorithm is proposed based on gradient profile sharpness. Gradient Profile Sharpness (GPS) is an edge sharpness matrix which is extracted from two gradient description models, i.e. a Gaussian mixture model for the description of different kind of gradient profile. The proposed approach will generate superior HR image with better visual quality, lower reconstruction error than the input image. To improve the HR image pixel quality, we will be use some filters. And compare the PSNR values to the input image.

Keywords - Super Resolution Single Image, Gradient Profile Sharpness, Profile dictionary.
\end{abstract}

\section{INTRODUCTION}

Image [1] Super Resolution (SR) is a process which artificially produces a high resolution (HR) image from one or more low resolution (LR) images. Image SR is utilized in various applications involving low cost, low resolution camera sensors to improve the resolution for better image analysis or to make the images visually appealing for the end user. Examples of such applications include medical imaging, satellite imaging, cell phone cameras, digital photography etc. Image processing literature contains a wide variety of SR algorithms which can be broadly Classified into multi image SR and single image SR. Multi image SR targets to estimate a HR image from several LR snapshots with sub-pixel shifts. Various single image SR algorithms appearing in the literature are mostly interpolation based or based on learning from a large training database of HR-LR pairs of natural images. The problem definition of the system is to detect Low Resolution (LR) patches from Input Images. Removal of additive white Gaussian noise by using various method and Quality of generated image is low which have less noisy image. Improve the quality and reconstruct the HR Image.

The remainder of this paper is organized as follows: Section II briefly reviews the related literature on single image SR. In Section III, we describe the proposed work of the Gradient Profile Sharpness method for single image SR. In Section IV, we describe details of experiments conducted on a set of natural images are given. Section V concludes the paper and future work.

${ }^{1}$ Department of Computer Science and Engineering, YCCE, Nagpur, Maharashtra, India

${ }^{2}$ Department of Computer Science and Engineering, YCCE, Nagpur, Maharashtra, India 


\section{RELATED LITERATURE}

In this section we will be discussing about the related literature. In Single Image Super Resolution Algorithm as have been discussed earlier have the involment of the various techniques and algorithm are discuss in below.

Min Chunn Yang et al. [1] presented a novel self-learning approach for SR. In this proposed framework, they advance a support vector regression (SVR) with image sparse representation, which offers excellent generalization in modelling the relationship between images and their associated SR versions. In most cases, this method quantitatively and qualitatively outperforms state -of the art and bicubic interpolation learning based SR approaches. Changhyun Kim et al. [2] presented an example-based super-resolution, it is difficult to determine appropriate highfrequency (HF) patches from database by using only the information of one input image. In this letter, they utilize the sharpness of high-resolution patch candidate for the reliable determination of HF patches. Experimental results shows that the proposed algorithm provide sharper detail compared to the existing algorithms. Shuyum yang et at. [3] Presented a single image superresolution reconstruction (SISR) via sparse coding has attracted increasing interest. In this paper, they proposed a multiple-geometric-dictionaries-based clustered sparse coding scheme for SISR. Firstly, a large number of High resolution (HR) image patche are randomly extracted from a set of example training images and clustered into several group of "geometric patches," from which the corresponding "geometric dictionaries" are learned to further sparsely code each local patch in a low resolution (LR) image. The HR residual image was then estimated from the recovered HR image and compensated to the recovered HR image to better preserve the subtle details of the images.

Jianchao Yang et al. [4] proposed a novel coupled dictionary training method for single-image super-resolution (SR) based on patch wise sparse recovery, where learned couple dictionaries are relate the low and high resolution (HR) image patch space via sparse representation. The learning process enforces that the sparse representation of a low-resolution (LR) image patches in terms of low resolution dictionary can well reconstruct its underlying HR image patch with the dictionary in high-resolution image patches space. The coupled dictionary training enforces that the sparse representation derived from the LR image patch quantitatively and qualitatively, our fast implementation could achieve the same level of performance as the original exact algorithm, but at a much higher speed. Jianzhou Feng et al. [5] present an Expected Patch Log Likelihood (EPLL) framework using Gaussian Mixture Model (GMM) prior for image restoration was recently proposed with performance comparable to the state-of-the-art algorithms. However, EPLL uses generic prior train from offline image patch. In this paper, they extend the EPLL framework to an adaptive one, named A-EPLL, which not only concerns with likelihood of restored patches, but also trains the GMM to fit for the degraded image. Experiment results show that A-EPLL outperforms the original EPLL significantly on several image restoration problems, like inpainting, deblurring and denoising. This new framework contains an efficient GMM learning algorithm, which lead to better prior than the predefined one. By further optimizing the initialization and parameter settings, the performance under different tasks are enhanced significantly.

Milad Niknejad et al. [6] presented an address the problem of recovering degraded images using multivariate Gaussian mixture model (GMM) as prior. The GMM framework in our method for image restoration is based on the assumption that the accumulation of similar patch in a neighborhood are derived from a multivariate Gaussian probability distribution with a specific covariance and mean. Previous method of image restoration with GMM have not considered 
spatial (geometric) distance between patches in clustering. Our method is highly comparable with the state-of-the-art methods, and our image interpolation method outperform previous stateof-the-art method. Michal Irani et al.[7] proposed an approach is similar to back-projection used in tomography. Examples of improved image resolution is given, with computation of the unknown image displacements Super resolution is shown to be feasible for image sequences, when the relative displacements can be computed accurately, and with approximate knowledge of the imaging process.

Michael Elad et al. [8] In this paper, they rederive these algorithms as approximations of the Kalman filter and then carry out thorough analysis of their performance. For each algorithm, they calculate a bound on its deviation from the Kalman filter performance. They also show that the propagated information matrix within the R-SD algorithm remain sparse in time, ensuring the applicability of this algorithm. The proposed algorithms assumes exact knowledge of the motion between image and the blur operator. This is also the assumption underlying the analysis they perform. Yonggun Lee et al.[9] In this paper, they presented a novel single image superresolution technique based on modelling embedding is proposed. Conventional modelling embedding technique uses gradient feature vector in modelling selection. However, gradient feature is high-frequency feature, it is not appropriate for feature representation in weak edge and texture region. Therefore, instead of using only gradient feature as in modelling embedding, hybrid feature which combine gradient features for edge regions and luminance norm feature for non-edge region is used. Results showed that strong edge was reconstructed as well as conventional methods and details in weak edge and texture were recovered better.

Radu Timofte et al.[10] they presented a fast super-resolution methods while making no compromise on quality. First, support the use of sparse learned dictionaries in combination with neighbor embedding methods. In this, the nearest neighbor is computed using the correlation with the dictionary atoms rather than the Euclidean distance. Second, show that using global collaborative coding has considerable speed advantages, reducing the super-resolution mapping to a precomputed projective matrix. Third, the anchored neighborhood regression. That is to anchor the neighborhood embedding of a low resolution patch to the nearest atoms in the dictionary and to precompute the corresponding embedding matrix. They obtain similar or improved quality and one or two order of magnitude speed improvements. Palakkattillam Sandeep et al.[11] In this paper, they present an Approach the single image SR problem by using a joint Gaussian mixture model learnt from concatenated vectors of high and low resolution patches sampled from a large database of pair of high resolution and the corresponding low resolution image. Covariance matrices of the learnt Gaussian model capture the inherent correlation between high and low resolution patches, which are utilized for inferring high resolution patch from given low resolution patch. The proposed joint GMM method can be interpreted as the GMM analogue of joint dictionary based algorithm for single image SR. As a result of the GMM prior assumed to model the patches, the overall SR algorithm becomes a piecewise linear filter involving $K$ linear filters, where, $K$ is the total number of Gaussian components included in the mixture.

Jianchao Yang et al.[12] In this paper, they presented a low-resolution image is viewed as down sampled version of a high-resolution images, whose patches are assumed to have a sparse representation with respect to an over-complete dictionary of prototype signal atom. The principle of compressed sensing ensures that under mild conditions, the sparse representation can be correctly recover from the down sampled signal. We will demonstrate the effectiveness of sparsity as a prior for regularizing the otherwise ill-pose super-resolution problems. We further 
show that a small set of randomly chosen raw patch from training image of similar statistical nature to the input image generally serve as a good dictionary, in the sense that the computed representations are sparse and the recovered high-resolution image is competitive or even superior quality to images produced by other SR methods. Jian Sun et al. [13] In this paper, they propose an image super-resolution approach using a novel generic image prior gradient profile patches, which is a parametric prior describing the shape and the sharpness of the image gradients. Using the gradient profile patches learned from a large number of natural images, they provide a constraint on image gradients when they estimate a hi-resolution image from a lowresolution image. With this simple but very effective prior, they were able to produce state-ofthe-art result. The reconstructed HI resolution image is sharp while has rare ringing or jaggy artifacts. One possible solution is to denoise the LR image first, then add the up-sampled noises back after the image super-resolution.

$\mathrm{Yu}$-Wing Tai et al. [14] image super resolution (SR) focuses on ways to remove edge artifacts in up sampled images. Under large magnification. However, texture region becomes blurred and appear homogenous, resulting in a super-resolution image that look unnatural. Alternatively, learning-based SR approaches use a large database of exemplar images for "hallucinating" detail. The quality of the up sampled image, especially about edges, is dependent on the suitability of the training images. They propose an approach to extend edge-directed super-resolution to include detail from an image/texture example provided by the user supplied example.

Xie Qinlan et al.[15] they presents a high frequency patches of low-resolution image to accelerate the procedure. Before the super-resolution, classify this method is used to mark the high-frequency patches of the low-resolution image with corresponding labels. During super resolution, the distances between each matching patches of low resolution image and middlefrequency patches of training data set are computed. The candidate patch is the patch within training set with the minimum distance. For the patch labelled with non-edges, few candidates are selected, while for flat patches the matching steps can be cancelled, directly replacing highresolution patches by enlarged interpolation of low-resolution patches. Method can reduces effectively the computational complexity.Jian Sun et al.

[16] they present an image gradients are represented by gradient profiles, which are 1-D profiles of gradient magnitudes perpendicular to image structures. They model the gradient profiles by a parametric gradient profile. Using this model, the prior knowledge of the gradient profiles are learned from a large collection of natural images, which are called gradient profile prior. Based on this prior, they propose a gradient field transformation to constrain the gradient fields of the high resolution image and the enhanced image when performing single image super-resolution and sharpness enhancement. With this very effective but simple approach, they are able to produce state-of-the-art results. The reconstructed high resolution images or the enhanced images are sharp while have rare ringing or jaggy artifacts. Fei Zhou et al. [17] they present an idea is using multisurface fitting to take full advantage of spatial structure information. Each site of lowresolution pixels is fitted with one surface, and the final estimation is made by fusing the multisampling values on these surfaces in the maximum posteriori fashion. With this method, the reconstructed high-resolution images preserve image details effectively without any hypothesis on image prior. Furthermore, they extend our method to a more general noise model. Experimental results on the simulated and real-world data show the superiorit of the proposed method in both quantitative and visual comparisons.

\section{PROPOSED WORK}


Image super resolution algorithm is proposed based on gradient profile sharpness (GPS) and Profile Dictionary. GPS is an edge sharpness matrix which is extracted from two gradient description models, i.e. a triangle model and a Gaussian mixture model for the description of different kind of gradient profile. The proposed approach will generate superior HR image with better visual quality, lower reconstruction error and compare the PSNR value of GPS method to input image.

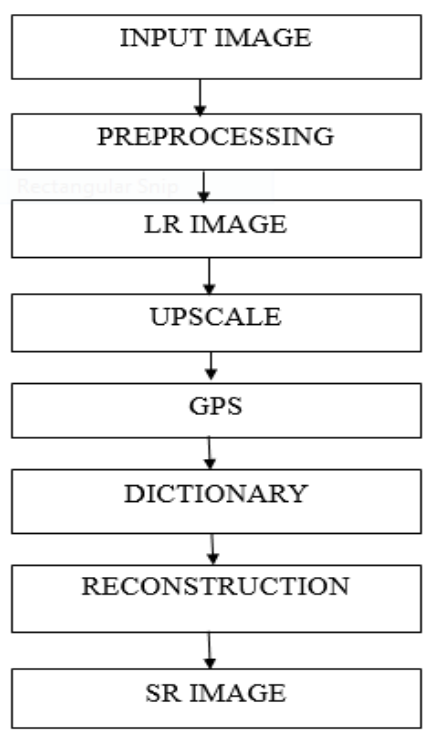

Fig.1 General Flow

In fig. 1 General Flow diagram of Super Resolution Reconstruction of Image Gradient Profile Sharpness. First we take Input Image is pre-processed it we get smooth image then converted into low resolution (LR) image upscale and patch it. Apply gradient profile sharpness matrix for the edge sharpness of input image. Create a dictionary which contain a pair of upscale and high resolution patches. With help of dictionary replace the patch from the output of gradient profile sharpness in which the best patch is replace it and obtained the sharp image and last reconstructed the super resoluted image which contain less reconstruction error as compare to the original image.

\section{PHASES OF GENERAL PROPOSED WORK:}

PHASE I:

1. Analysis of literature data

2. Searching of Data Set

PHASE II:

3. Preprocessing

4. Upscale the patch

5. Create Dictionary

PHASE III:

6. Reconstruct the LR in HR with high pixel quality.

7. Super Resolution single image is formed. 


\section{EXPERIMENTAL RESULTS}

In this section we are briefly describe the live database [18]. For the construction of training data, we randomly chose images on a public website. The experiment is performed on a PC running a single core of Intel Pentium 3.0 GHz CPU. We are using the MATLAB 13a for execute it.

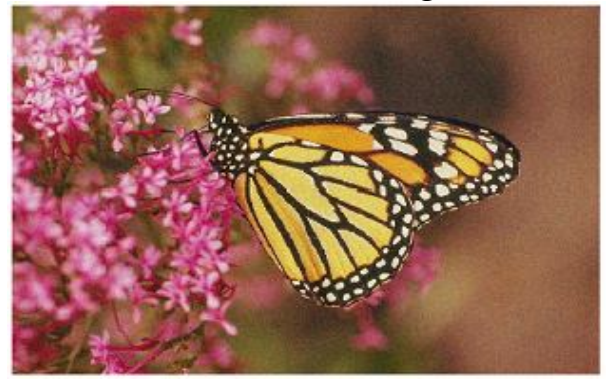

Fig. 2 Original Image

In Fig. 2 Original Image, we are simply taking an image from live database and perform further operation.

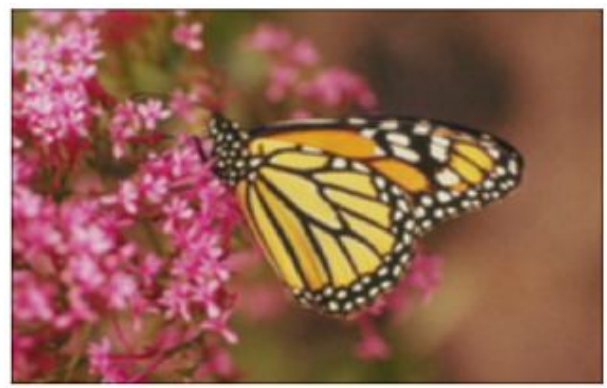

Fig. 3 Weiner Filtered Image

In Fig. 3 Weiner filtered Image, here we are applying wiener filter to smooth an image as in the original resolution of image.

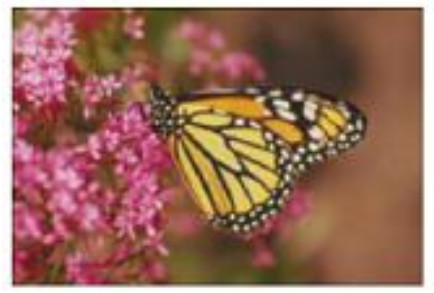

Fig. 4 Low Resolution Image

In Fig. 4 Low Resolution Image, we apply a resize function on the original image and obtained a low resoluted image as output. Which of half of the size of input image.

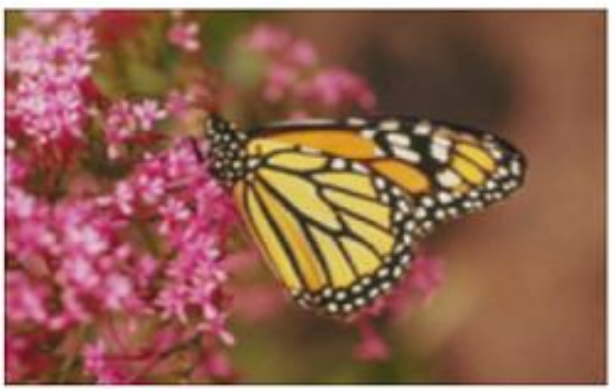

Fig. 5 Upscale Image 
In Fig. 5 Upscale Image, here we obtained an upscale image for the use of creating a dictionary. Which is of same resolution of the input image.

\section{CONCLUSION AND FUTURE WORK}

In this paper, Single Image super Resolution Reconstruction using gradient Profile Sharpness is discuss based on the edge sharpness matrix i.e. triangle model and Gaussian mixture model. Super resolution is the fundamental research area in image processing and overcome the resolution problems of imaging systems. An interesting point finding from the above survey is that, since different SR methods have been developed for different applications using different model parameters and assumptions. And also execute the further process i.e. apply wiener filter for smoothening image and after that converted into low resolution image then upscale it with the help of input image. In Future Work, single image super resolution with high PSNR value and output of an image will be sharp, high quality pixel will be obtained.

\section{REFERENCES}

[1] Min-Chun Yang and Yu-Chiang Frank Wang, Member, "A Self-Learning Approach to Single Image SuperResolution", IEEE Transactions On Multimedia., Vol. 15, No. 3, April 2013

[2] Changhyun Kim, Kyuha Choi,Jong Beom Ra," Example-Based Super-Resolution via Structure Analysis of Patches", IEEE Signal Processing Letters, Vol. 20, No. 4, April 2013

[3] Shuyuan Yang, Member, Min Wang, Yiguang Chen, and Yaxin Sun, "Single-Image Super-Resolution Reconstruction via Learned Geometric Dictionaries and Clustered Sparse Coding", IEEE Transactions on Image Processing, Vol. 21, No. 9, September 2012

[4] Jianchao Yang, Zhaowen Wang,Zhe Lin, Scott Cohen, and Thomas Huang, Life Fellow, "Coupled Dictionary Training for Image Super-Resolution”,IEEE Transactions On Image Processing, Vol. 21, No. 8, August 2012

[5] Jianzhou Feng, Li Song,Xiaoming Huoy, "Image Restoration Via Efficient Gaussian Mixture Model Learning”, IEEE 2013.

[6] Milad Niknejad, Hossein Rabbani, Massoud Babaie-Zadeh, "Image Restoration Using Gaussian Mixture Models With Spatially Constrained Patch Clustering",IEEE Transactions On Image Processing, Vol. 24, No. 11, November 2015

[7] M. Irani and S. Peleg, "Super resolution from image sequences," in Proc.10th International Conference Pattern Recognition, pp. 115-120 Jun. 1990.

[8] M. Elad and A. Feuer, "Superresolution reconstruction of image sequences," IEEE Trans. Pattern Anal. Mach. Intell., Vol. 21, No. 9,pp. 817-834, Sep. 1999.

[9] Yonggun Lee, Yoonsik Choe," Neighbor Embedding Based Single Image Super-Resolution using Hybrid Feature and Adaptive Weight Decay Regularization," pp.185-187, IEEE 2014.

[10] Radu Timofte, Vincent De Smet, and Luc Van Gool," Anchored Neighborhood Regression for Fast ExampleBased Super-Resolution," precessiding on International Conference on Computer Vision pp.1920-1927, IEEE 2013.

[11] Palakkattillam Sandeep, Tony Jacob," Single Image Super-Resolution Using a Joint GMM Method," IEEE TRANSACTIONS ON IMAGE PROCESSING, VOL. 25, NO. 9, SEPTEMBER 2016.

[12] Jianchao Yang, John Wright,"Image Super-Resolution as Sparse Representation of Raw Image Patches,"2008 IEEE.

[13] Jian Sun,” Image Super-Resolution using Gradient Profile Prior,"2008 IEEE.

[14] Yu-Wing Tai," Super Resolution using Edge Prior and Single Image Detail Synthesis,"2010 IEEE.

[15] Xie Qinlan," Improved Example-based Single-image Super-resolution," In proceeding of 2010 3rd International Congress on Image and Signal Processing (CISP2010).

[16]Jian Sun," Gradient Profile Prior and Its Applications in Image Super-Resolution and Enhancement," IEEE TRANSACTIONS ON IMAGE PROCESSING, VOL. 20, NO. 6, JUNE 2011

[17] Fei Zhou, Wenming Yang," Interpolation-Based Image Super-Resolution Using Multisurface Fitting," IEEE TRANSACTIONS ON IMAGE PROCESSING, VOL. 21, NO. 7, JULY 2012

[18]Live Database for Image Quality Assessment.[Online].Available:http://live.ece. Utexas.edu/research/quality 